\title{
LEGITIMACIÓN DE LA POLICÍA, UN ESTUDIO SOBRE POLÍTICAS DE SEGURIDAD, PARTICIPACIÓN E INTEGRACIÓN CIUDADANA EN BOLIVIA
}

Legitimation of the Police: a study on security policies, participation and citizen integration in Bolivia

Legitimação da polícia: estudo sobre políticas de segurança, participação e integração do cidadão na Bolívia

Received: feb./2020

Accepted: feb./2020

Available online: mar. $/ 2020$

Juan Carlos Poveda Velasco, Magister, Universidad Mayor, Real y Pontificia de San

Francisco Xavier de Chuquisaca, Bolivia. E-mail: juancapoveda@gmail.com.

https://orcid.org/0000-0002-6279-899X

José Luis Assaf Irahola, Coronel de Polícia, Policia Nacional, Bolivia. jassafi@hotmail.com.

Resumo: La inseguridad ciudadana es uno de los principales problemas del mundo, la Policía Boliviana tiene el rol constitucional de preservar el orden y seguridad ciudadano, causando sus métodos y prácticas de los últimos años un fuerte cuestionamiento de la sociedad en su conjunto, por ello la jerarquía policial ha visto como necesario identificar políticas, que desde la participación ciudadana e institucional, permita devolver la legitimidad a la Policía al mismo tiempo que se reduzca la incidencia de delitos. En ese sentido, la presente investigación tiene como objetivo identificar políticas de Seguridad Ciudadana que contribuyan a la legitimación del rol constitucional de la Policía Boliviana incluyendo participación e integración ciudadana; para ello se ha recurrido a consultas a expertos en el diseño, implementación y evaluación de políticas de seguridad ciudadana, de manera que a partir de las conceptualizaciones teóricas actuales se bosqueje una estructura de políticas de seguridad ciudadana que puedan ser implementadas en la Policía Boliviana. 
Palabras clave: Seguridad ciudadana, Policía, Políticas de Seguridad, Administración Pública.

\begin{abstract}
Citizen insecurity is one of the main problems of the world, the Bolivian Police has the constitutional role of preserving citizen order and security, causing its methods and practices of recent years a strong questioning of society as a whole, hence the hierarchy The police have seen the need to identify policies that, through citizen and institutional participation, allow police to return legitimacy while reducing the incidence of crime. In this sense, the present investigation has the objective of identifying Citizen Security policies that contribute to the legitimation of the constitutional role of the Bolivian Police, including citizen participation and integration; For this reason, experts have been consulted in the design, implementation and evaluation of citizen security policies, so that from the current theoretical conceptualizations a structure of citizen security policies can be drawn up that can be implemented in the Bolivian Police.
\end{abstract}

Keywords: Citizen Security, Police, Security Policies, Public Administration.

Resumo: A insegurança cidadã é um dos principais problemas do mundo, a Polícia Boliviana tem o papel constitucional de preservar a ordem e a segurança dos cidadãos, causando aos seus métodos e práticas dos últimos anos um forte questionamento da sociedade como um todo, portanto, a hierarquia A polícia considerou necessário identificar políticas que, a partir da participação cidadã e institucional, permitam que a legitimidade seja devolvida à polícia e reduza a incidência de crimes. Nesse sentido, a presente investigação visa identificar políticas de Segurança Cidadã que contribuam para a legitimação do papel constitucional da Polícia Boliviana, incluindo a participação e a integração do cidadão; Para isso, especialistas foram consultados sobre o desenho, implementação e avaliação de políticas de segurança cidadã, de modo que, a partir das conceituações teóricas atuais, seja apresentada uma estrutura de políticas de segurança cidadã que possa ser implementada na Polícia Boliviana.

Palabras-chave: Segurança Pública, Polícia, Políticas de Segurança, Administração Pública.

\title{
INTRODUCCIÓN
}

La inseguridad ciudadana es uno de los principales problemas del mundo. En las últimas décadas, este fenómeno ha mostrado un incremento, generalización y diversificación que conlleva a altos impactos sociales. En Latinoamérica, el crecimiento de la población ha generado un aumento de los delitos y una percepción de inseguridad según el Informe Regional de Desarrollo Humano, pues en la primera década del 2000 la tasa de homicidios creció un 11 \% y fallecieron más de un millón de personas a causa de la violencia criminal. Así mismo, los robos se han casi triplicado en los últimos 25 años. Además, en 2012 casi medio millón de personas perdieron la vida a causa de homicidios dolosos en el mundo, y más de una tercera parte de estos tuvieron lugar en el continente americano (36\%), seguidos de África, Asia, 
Europa y Oceanía con $31,28,5$ y $0,3 \%$, respectivamente. El homicidio doloso, junto con otros delitos violentos, es una amenaza considerable para la población, ya que su impacto, además de la pérdida de vidas humanas, genera miedo e incertidumbre (Paez Murillo, Peón Escalante, \& Ramírez, Pedraza, 2018; PNUD, 2018; Vidales Rodríguez, 2010).

Los países latinoamericanos han experimentado delitos de carácter transnacional, como el tráfico de personas, armas, drogas ilícitas y terrorismo, los cuales rebasan su capacidad y contribuyen a la confusión entre las funciones que le competen a las agencias civiles y militares encargadas de proporcionar seguridad. Por lo anterior, este panorama obliga a los estados a dirigir la mirada hacia una agenda de programas y políticas de seguridad y desarrollo en un contexto más específico, en especial, el de seguridad ciudadana (Hernández-Baqueiro \& Suárez-González, 2015; Medina Ariza, 2010; Paez Murillo et al., 2018; PNUD, 2018).

Actualmente la inseguridad representa el principal reto para las autoridades, en los distintos niveles de gobierno. El tema es particularmente sensible para los municipios con alta concentración urbana, pues son la instancia más cercana al ciudadano. Ese ciudadano que reclama mayor seguridad en las calles y en su hogar (Alvarez, 2005; Belaunde de Cárdenas, 2015; Frühling, 2012; PNUD, 2018). La inseguridad ciudadana es percibida por la población como uno de los principales problemas de América Latina, al igual que en Bolivia.

Adicionalmente, el debate sobre el rol de la policía en las políticas de seguridad de las ciudades se centró durante varias décadas en la disyuntiva represión-prevención, los temas sobre la seguridad interna tienen un tenor diferente, las políticas de seguridad más recientes y exitosas se caracterizan por ser esencialmente preventivas, de manera que hacen de la policía un actor importante que debe intervenir en el restablecimiento de la seguridad entre los ciudadanos. La disyuntiva mencionada ha perdido fuerza en favor de otras premisas como disuasión-asociación (prevención), que apuntan a restablecer el tejido social como piedra angular de lucha contra 
inseguridad (Hernández-Baqueiro \& Suárez-González, 2015; Paez Murillo et al., 2018; PNUD, 2018; Ruiz Vasquez, 2003; Vidales Rodríguez, 2010).

La evolución del concepto de seguridad, al igual que sus interpretaciones, propicia nuevas y mejores prácticas, con un enfoque complejo que permite no perder de vista el potencial de las dimensiones humanas y que muestra que la verdadera riqueza de las naciones son las personas, razón por la cual el objetivo del desarrollo debe ser propiciar condiciones que permitan una vida larga, saludable y creativa que incluya a todos los actores relacionados con la problemática, estatales y civiles (Acero, 2005; Alvarez, 2005; Paez Murillo et al., 2018; Salgado Ibarra, 2010; Sozzo, 2012).

Por otro lado, en sociedades democráticas, la policía, como cualquier otra institución, necesita legitimidad para operar y cumplir con sus cometidos, así como para mantenerse en el tiempo y desarrollarse - las leyes le dan legalidad, pero la sociedad es quién legitima - en el caso específico de la policía, que para el cumplimiento de sus funciones puede llegar a hacer uso de la fuerza, esta afirmación es especialmente relevante (Paez Murillo et al., 2018; Requena Hidalgo, 2016; Ruiz Vasquez, 2003).

Con las consideraciones previas se plantea el siguiente problema: ¿Cuáles son las políticas de Seguridad Ciudadana, que incluyan participación e integración ciudadana, que contribuyan a la legitimación del rol constitucional de la Policía Boliviana?

Con el convencimiento de que es necesario profundizar en el debate académico, público, político y profesional sobre las posibilidades que tiene el trabajo policial de promover comportamientos que respeten las normas y las leyes, así como la colaboración ciudadana con la propia policía, de manera que se reduzca la delincuencia al mismo tiempo que se devuelve la legitimidad al accionar de la institución policial, el presente trabajo de investigación se justifica por ser un tema de alta importancia para la sociedad, es actual y pertinente, así como representa un aporte a la academia, en el contexto del problema se plantea el siguiente objetivo: Identificar 
políticas de Seguridad Ciudadana que contribuyan a la legitimación del rol constitucional de la Policía Boliviana incluyendo participación e integración ciudadana.

Revisión bibliográfica

\section{Delincuencia y Seguridad Ciudadana}

La delincuencia ha estado presente en la sociedad humana, sin embargo, a partir de 1990, década en la que la UNICRI (United Nations Interregional Crime and Justice Research Institute) compila la información sobre esta área a nivel mundial, es imposible no reconocer el nuevo fenómeno de la delincuencia y violencia que afecta la casi totalidad de las ciudades del mundo y su cambio de dimensión como su mayor diversidad en las áreas urbanas que hacen de este fenómeno una crisis de sociedad (Hernández-Baqueiro \& Suárez-González, 2015). Por consiguiente, no se puede eludir una respuesta sin poner en tela de juicio algunas de las funciones e instituciones fundamentales de los gobiernos, considerados como responsables de la seguridad, como también los procesos deconstrucción de la cohesión social (HernándezBaqueiro \& Suárez-González, 2015; Paez Murillo et al., 2018; Vidales Rodríguez, 2010).

Múltiples son los intentos de justificación de este descontrol mundial en la delincuencia, pero se encuentra relación que el desarrollo constante de la sociedad de consumo es acompañado así por el desarrollo de una delincuencia de apropiación que constituye una suerte de justificación social para la redistribución violenta operada generalmente por los que están, si no totalmente excluidos, al menos en los márgenes de esta sociedad, la modernidad se caracteriza pues también por esta competencia siempre más cruel por la posesión de los bienes de consumo, en una sociedad donde el anonimato y la deslegitimación institucional facilita la tarea de los ladrones(Constantino, 2014; Hernández-Baqueiro \& Suárez-González, 2015; Medina Ariza, 2010). 
En este contexto en los últimos años, con el aumento de la conflictividad social y la delincuencia, asociada a las consecuencias de la crisis y desigualdades económicas, las intervenciones policiales en las manifestaciones de dicha conflictividad conllevan a la reflexión sobre la legitimidad de la institución como una cuestión verdaderamente pertinente y necesaria. En Bolivia, Latino América y el Mundo, la institución policial es duramente criticada por la influencia que tienen los grupos delincuenciales sobre su actuar (Frühling, 2012; García Gallegos, 2013; Hernández-Baqueiro \& Suárez-González, 2015; Requena Hidalgo, 2016).

\section{Legitimidad policial y seguridad ciudadana}

La seguridad está hoy en la agenda de todos los debates en América Latina. Varias encuestas de opinión muestran que la falta de seguridad es una de las preocupaciones mayores de los habitantes de la región, en cerrada competencia con el desempleo y la educación. El auge de la delincuencia generalizado y exponencial como inexplicado constituye un aumento normal de lo que era hasta entonces un fenómeno marginal, sino una crisis de sociedad, porque indica el surgimiento de una forma de anomia, donde las reglas de convivencia no son compartidas por todos los grupos sociales. Reconstruir consensos parece más importante en este caso que la simple contención de la violencia o de los actos en conflicto con la ley (Hernández-Baqueiro \& Suárez-González, 2015; Paez Murillo et al., 2018; Ruiz Vasquez, 2003).

Este aumento, fenómeno relativamente reciente en la región, tiene ya confirmados efectos negativos en el desarrollo humano y se presenta también como un elemento distorsionador para la dinámica democrática. La violencia y la inseguridad son en el estado actual de cosas, un condicionante para el desarrollo, así como la progresiva erosión de la credibilidad en las instituciones democráticas, que se ven superadas por un fenómeno para el que no parecen tener respuesta (Alvarez, 2005; Paez Murillo et al., 2018; Torres Angarita, 2010; Vidales Rodríguez, 2010). 
La legitimidad, definida como el derecho a gobernar y el reconocimiento de ese derecho por parte de los gobernados, sobre todo en lo relacionado con la "legitimidad policial" se convierte en un factor estratégico de las políticas públicas de seguridad, pues es la base de la conformidad con las leyes y muy especialmente con las disposiciones, las indicaciones y las órdenes que la propia policía establece. A mayor legitimidad de la policía, mayor disposición por parte de la ciudadanía a aceptar y a cumplir sus órdenes, mayor disposición a colaborar activamente con ella y mayor disposición a cumplir con las leyes en general. Por el contrario, a menor legitimidad policial, cabe esperar una menor deferencia hacia la policía y sus agentes, una menor colaboración con ellos y una menor predisposición al cumplimiento voluntario de las normas, lo que conduce a escenarios de gobernabilidad más difíciles, las que resultan ser el ambiente propicio para el desarrollo y crecimiento de la delincuencia (Alvarez, 2005; Constantino, 2014; Jaime \& Torrente, 2017; Medina Ariza, 2010; Requena Hidalgo, 2016).

En este estado situacional es indispensable redimensionar el rol constitucional de la Policía Boliviana, a través de identificar Políticas de Seguridad Ciudadana que al mismo tiempo de incorporar la participación activa de la ciudadanía permitan legitimar - reconocimiento y cumplimiento - su accionar.

\section{Seguridad Pública y Ciudadana}

De acuerdo a lo expuesto por (Alvarez, 2005; Constantino, 2014; Frühling, 2012; Requena Hidalgo, 2016; Salgado Ibarra, 2010), existe consenso entre juristas e investigadores que las políticas de seguridad pública y ciudadana se encuentran en el marco de las constituciones y la legislación penal de los países, el “continuum” de la normativa jurisdiccional de manera general comprenden las siguientes fases o etapas:

- Prevención del delito. Ámbito de acción esencialmente de las policías de orden y de tránsito, gobiernos locales y secretarias departamentales de seguridad ciudadana. 
- Procuración de justicia. Ámbito de competencia de la procuraduría general, investigación policial, servicios periciales y Ministerio Público (físcalía).

- Administración de justicia. Que incorpora a los tribunales de justicia.

- Ejecución de sentencia. Competencia del sistema penitenciario.

En este ámbito, las políticas de seguridad ciudadana se encuentran circunscritas en la fase de Prevención del delito, sin embargo, se debe tener un concepto integral de cada una de estas fases o etapas, pues sino existe un adecuada coordinación entre las diferentes instituciones que aportan a este "continuum", se generarán incentivos negativos hacia la labor de las policías preventivas e investigativas, quienes en última instancia son las protagonistas de la instrumentación de las políticas de seguridad ciudadana (García Gallegos, 2013; Requena Hidalgo, 2016; Ruiz Vasquez, 2003; Salgado Ibarra, 2010).

\section{Políticas de Seguridad Ciudadana y la Institución Policial}

Según (Alvarez, 2005; Constantino, 2014; Jaime \& Torrente, 2017; Medina Ariza, 2010; Requena Hidalgo, 2016), la política de seguridad ciudadana se define comúnmente como el conjunto de las acciones gubernamentales destinadas a prevenir, investigar y castigar los delitos en la jurisdicción de la seguridad ciudadana y los delitos vinculados. La literatura especializada tiende a clasificar las políticas de seguridad de los gobierno de turno según un criterio ideológico, acercando a modelos seguritarios de izquierda, centro o de derecha. Estos modelos de criterios ideológicos suelen tener como dimensiones: a) la forma de gobierno del sistema de seguridad y b) las acciones a emprender para: i) prevenir el delito, ii) investigar y enjuiciar a los presuntos delincuentes y iii) castigar a los criminales.

Los modelos académicamente aceptados plantean los siguientes paradigmas - izquierda, centro y derecha - considerando las dimensiones de aplicación de la política de seguridad (Alvarez, 
2005; Constantino, 2014; Jaime \& Torrente, 2017; Medina Ariza, 2010; Requena Hidalgo, 2016):

- Las políticas de seguridad situadas cerca de la izquierda se caracterizan por promover un modelo participativo de dirección y control de la política de seguridad ciudadana; implementan mecanismos de participación de la ciudadanía en la elaboración y en la implementación de la política de seguridad: funcionarios civiles a cargo del ministerio y de la policía, foros de seguridad, audiencias públicas, defensores públicos contra la inseguridad y el maltrato policial, entre otras.

- Las políticas seguritarias de la derecha, sostienen que el gobierno del sistema penal debe estar en manos de miembros o representantes de esta burocracia o de las fuerzas armadas. Es decir, de especialistas en el manejo de la violencia legítima. Más allá de los distintos factores que afectan la comisión de delitos, consideran que el sistema de seguridad es un elemento clave y necesario, pues siempre existen en las sociedades, por más igualitarias que sean, gente que ejerce violencia arbitraria sobre la integridad física y los bienes de otras personas o de todos.

- En el medio de estos dos paradigmas de políticas de seguridad se encuentran los discursos de centro, que combinan de manera más o menos coherente argumentos y políticas de derecha y de izquierda. De hecho, muchos políticos y actores sociales relevantes proponen acciones integrales para mejorar la situación de los sectores más vulnerables y marginales, a la vez que señalan la importancia de políticas punitivas que castiguen a aquellas personas que a pesar del esfuerzo del Estado, deciden delinquir.

\section{Seguridad ciudadana y espacio defendible}

La teoría del espacio defendible es generadora de políticas de seguridad relacionadas con la participación comunitaria y la vigilancia territorial. El espacio defendible es un modelo para 
ambientes residenciales que inhibe el delito por medio de la creación de la expresión física de una comunidad social que se defiende a sí misma (Medina Ariza, 2010; Newman, 1972; Vidales Rodríguez, 2010).

El empleo de cámaras de vigilancia enlazadas con circuitos cerrados de televisión (CCTV) se ha convertido en una tecnología de control que sirve numerosas funciones hoy en día tanto en espacios privados como públicos. La vídeo-vigilancia es una herramienta de prevención situacional que sirve para incrementar el nivel de vigilancia formal y, por tanto, tiene un papel disuasorio al aumentar la percepción subjetiva de los delincuentes de que pueden ser identificados y detenidos (Hernández-Baqueiro \& Suárez-González, 2015; Ruiz Vasquez, 2003; Salas Torres, 2015).

\section{Policía de Intolerancia Cero - teoría de los cristales rotos}

Esta teoría fue adoptada por William Bratton, el jefe de la policía de Nueva York elegido por Rudolph Giuliani a principios de los 90. Bratton contaba entre sus consejeros con George Kelling y su liderazgo se caracterizó por la adopción de políticas en consonancia con las ideas de la teoría de los cristales rotos (Medina Ariza, 2010; Paez Murillo et al., 2018; Requena Hidalgo, 2016; Ruiz Vasquez, 2003).

La teoría de los cristales rotos propone que la existen de un vínculo causal entre la existencia de signos de desorden y la delincuencia. De acuerdo con esta teoría una sociedad que tolera el desorden social, las que considera como ofensas contra la calidad de vida, tales como la prostitución, la mendicidad agresiva, el consumo de drogas o alcohol en la calle, envía el mensaje a los potenciales delincuentes de que nadie toma responsabilidades por lo que pasa en la calle y de que se van a salir con la suya. La policía basada en la teoría de los cristales rotos propone, por tanto, la persecución agresiva de actos de desorden social, incluso si estos actos tan solo constituyen, desde el punto de vista legal, infracciones administrativas o delictivas de 
tipo menor (Medina Ariza, 2010; Newman, 1972; Torres Angarita, 2010; Vidales Rodríguez, 2010).

\section{Metodología}

La investigación se encuentra enmarcada en el ámbito de las Ciencias Sociales, intentando establecer desde la experiencia personal e institucional de operadores de políticas de seguridad ciudadana cuáles son las mejores políticas que se adapten a la sociedad boliviana y posibiliten una mayor legitimación del accionar de la Policía Boliviana (Chalmers, 2000; Morles, 2002; Sabino, 1992).

La investigación es de tipo aplicada pues pretende contribuir a la comprensión de los componentes relacionados con la seguridad ciudadana y la legitimidad del accionar de la Policía en general y de la Policía Boliviana en particular (Chalmers, 2000; Morles, 2002; Sabino, 1992). Es cualitativa, porque se analiza y sistematiza la experiencia de especialistas en el diseño, implementación y evaluación de políticas de seguridad ciudadana (Chalmers, 2000; Morles, 2002; Sabino, 1992).

Es descriptiva porque aportará información para entender mejor las características que definen las políticas actuales sobre seguridad ciudadana (Chalmers, 2000; Morles, 2002; Sabino, 1992). La descripción causa - efecto de las variables será abordada desde un criterio no experimental, pues se describirán las experiencias de directivos de operadores institucionales de políticas de seguridad ciudadana (Chalmers, 2000; Morles, 2002; Sabino, 1992).

Es de carácter inductiva, pues a partir de experiencias particulares se elabora un conjunto de políticas de seguridad ciudadana a ser sugeridas para su implementación en la Policía Boliviana (Chalmers, 2000; Morles, 2002; Sabino, 1992).

Se recurrió a la consulta de expertos como instrumento para la construcción y posterior validación de la estructura de políticas de seguridad ciudadana a ser sugeridas para su 
implementación a la Policía Boliviana (Morles, 2002; Robles Garrote \& Rojas, 2015; Sabino, 1992).

- Para la identificación de las políticas de seguridad ciudadana, se realizaron entrevistas a:

- Diez y ocho (18) jefes policiales, dos por cada Departamento geográfico de Bolivia, encargados de la Fuerza Especial de la Lucha Contra el Crimen (9) y de la Fuerza Especial de Lucha contra la Violencia (9).

- Nueve (9) responsables municipales de Seguridad Ciudadana, en las nueve capitales de Departamento de Bolivia.

- Nueve (9) presidentes de juntas vecinales de las capitales de cada Departamento de Bolivia.

- Para la validación de las políticas de seguridad ciudadana, se realizaron entrevistas a:

- Nueve (9) comandantes departamentales de la Policía.

- $\quad$ Nueve (9) directores municipales de seguridad ciudadana.

- Un (1) viceministro de seguridad ciudadana del Estado Plurinacional de Bolivia.

Resultados, análisis y discusión

La institución policial debe esforzarse por reducir la distancia entre su accionar y la confianza de la población, las reformas policiales deben estar relacionadas con la orientación a la comunidad y la proximidad, deben ser entendidas en el marco de un proyecto de mejora de su eficacia a través del reforzamiento de la legitimidad de la institución, por medio del acercamiento a la población a la que debe servir logrando su colaboración y confianza (Acero, 2005; Alvarez, 2005; García Gallegos, 2013; Requena Hidalgo, 2016).

En estos últimos años, con el aumento de la conflictividad social asociada a las consecuencias de la crisis económica y las intervenciones policiales en las manifestaciones de dicha 
conflictividad, la reflexión sobre la legitimidad de la institución se revela como una cuestión verdaderamente pertinente y necesaria. En términos generales, la legitimidad policial se centra en un conjunto específico de relaciones: las que hay entre los individuos, la policía, y el estado (Acero, 2005; Constantino, 2014; Frühling, 2012; Requena Hidalgo, 2016).

En la complejidad de las relaciones de la sociedad actual, la institución policial está sometida de forma creciente a un mayor número de presiones y demandas, las fuerzas de seguridad se enfrentan a esta realidad con singular intensidad dada su sobreexposición al entorno. La consecuencia es que la interacción diaria se ve afectada, así como la propia estructura interna de la Policía. Desde la perspectiva de las políticas de seguridad ciudadana, las fuerzas policiales son actores con funciones institucionales en un escenario sistémico, pero también son entes corporativos que aspiran a ubicarse en el entramado institucional como actores influyentes en la gobernanza social (Constantino, 2014; Jaime \& Torrente, 2017; Ruiz Vasquez, 2003). Esta posición privilegiada de la Policía entre los niveles político-institucionales y la ciudadanía le permite influir sobre el desarrollo, implementación y ejecución de las reformas en las políticas públicas y de seguridad ciudadana.

La garantía de la seguridad y la convivencia pacífica a través del establecimiento de normas para regularla es, más que una mera función del Estado, una función constitutiva del mismo. Esta garantía requiere evitar o minimizar la desviación respecto de dichas normas, las cuales, de algún modo, expresan una determinada idea del orden social. En la teoría política clásica, la facultad del Estado para imponer dicho orden sería el resultado de un contrato, el llamado contrato social. Según este contrato, el Estado aseguraría a la ciudadanía ciertos beneficios públicos en forma de derechos a cambio de que los individuos renunciasen a ciertas prerrogativas (Jaime \& Torrente, 2017; Newman, 1972; Requena Hidalgo, 2016; Sozzo, 2012; Vidales Rodríguez, 2010). 
El respeto por la Ley tiene que ver con los valores morales o éticos en los que se fundamentan las creencias individuales sobre sobre lo que está bien y lo que está mal, sobre los comportamientos considerados adecuados (Hernández-Baqueiro \& Suárez-González, 2015; Requena Hidalgo, 2016). La percepción de consistencia, de coherencia entre los propios valores y la Ley y el funcionamiento de las instituciones del sistema penal, como la policía, es un factor fundamental en la explicación del comportamiento individual en este ámbito. Todo ello, referido a las autoridades en general, también es aplicable a la policía. Las personas respetan la Ley y colaboran con la policía en su trabajo de hacer cumplirla porque creen que es lo que deben hacer (Medina Ariza, 2010; Ruiz Vasquez, 2003). Evitan comportarse de determinadas maneras, aunque no sea ilegal, incluso en contra de sus propios intereses, porque sus principios morales les llevan a ello.

Las políticas de seguridad tradicionalmente se han basado, sobre todo, en la articulación de un control policial eficaz y en el establecimiento de sanciones y penas lo suficientemente duras como para disuadir a la población de no cometer infracciones legales. Lógicamente, la clave del éxito de dichas políticas reside en la capacidad de crear y mantener esa amenaza disuasoria constante. Sin embargo, la realidad pone en cuestión la eficiencia de esta estrategia de control social a la hora de promover la conformidad de la ciudadanía con la Ley y la colaboración ciudadana con la policía (Alvarez, 2005; Belaunde de Cárdenas, 2015; Frühling, 2012; Gabaldón, 2013). Las reformas a las políticas de seguridad ciudadana deben considerar al mismo tiempo la eficacia del actuar de la policía al mismo tiempo de comprometer la conformidad de la ciudadanía, incorporando en este proceso un acercamiento entre la institución policial, las instituciones de la sociedad y a la sociedad civil.

En ese sentido las políticas de seguridad deben ser abordadas desde diferentes perspectivas y de manera integral, priorizando algunas áreas de intervención en coordinación con diferentes niveles del estado y la sociedad a nivel nacional, regional y local, en concordancia con los 
estudios de (Alvarez, 2005; Belaunde de Cárdenas, 2015; Frühling, 2012; Gabaldón, 2013; Jaime \& Torrente, 2017; Medina Ariza, 2010; Requena Hidalgo, 2016; Torres Angarita, 2010; Vidales Rodríguez, 2010), las áreas de intervención propuestas relacionadas con el accionar de la policía boliviana, son:

- Prevención social, con énfasis en programas que se dirigen hacia la juventud en riesgo;

- Prevención situacional, que cubre principalmente intervenciones urbanas;

- Actividades disuasivas de la policía;

- Fortalecimiento institucional.

\section{Politicas de Prevención social}

Las políticas de prevención social, pretende mejorar la calidad de vida de la sociedad a partir políticas integrales esencialmente preventivas y con énfasis en programas que se dirigen hacia la juventud en riesgo.

- Reducir la exposición a las oportunidades de conducta de riesgo. Orientada a centros de educación primaria y secundaria. Desarrollando actividades que incorporen la participación pasiva y activa de niños, adolescentes y jóvenes.

- Incluir a la población civil en la prevención del delito. Incorporando a juntas vecinales, organizaciones gremiales, asociaciones profesionales y otros, en el análisis de la problemática delictiva así como los factores que la propician; crear difusores civiles de seguridad ciudadana que operen en su ámbito social inmediata y mediata; elaboración y difusión de material informativo con participación civil y concordante con las costumbres de la población objetivo.

- Promover la solidaridad poblacional hacia la prevención del delito. Incorporar a las organizaciones sociales, organizaciones barriales, asociaciones juveniles y similares como scouts, rotary, etc., en intervenciones orientadas a la reducción de las causas que 
producen el malestar de los individuos, situaciones de abandono, sensibilización al consumo de drogas y alcohol, mendicidad extrema.

\section{Politicas de Prevención Situacional}

Cuyo fin es promover un vecindario seguro a través de la gestión de espacios residenciales y laborales, incorporando la participación poblacional e institucional, para la prevención del delito.

- Alterar el espacio físico. Implementando control de accesos, reductores de velocidad para vehículos, aumento de la vigilancia comunitaria, identificación de los puntos calientes.

- Cambios en la gestión del espacio físico. Aumentar la presencia de la policía especialmente cerca de los puntos calientes, implementación de sistemas electrónicos de vigilancia, incorporar a los residentes en la prevención del delito, creación de comités de vigilancia residencial.

- Cambios en los usos. Aumentar el uso cultural y deportivo de los espacios en diferentes momentos del día y de la noche, aumentar la variedad de los usos comerciales.

\section{Actividades disuasivas de la policía}

Relacionadas directamente con la institución policial y sus ámbitos de intervención.

- Patrullaje policial general y selectivo, especialmente en puntos calientes.

- Intervenir en grupos sociales con tendencia delictiva, pandillas juveniles especialmente controlando sus actividades al mismo tiempo que sean motivados a participar de actividades académicas, culturales y deportivas.

- Investigación de delitos incorporando organizaciones sociales y académicas. 


\section{Fortalecimiento institucional}

Dirigido a mejorar la efectividad con la que se ejecutan programas de seguridad ciudadana.

- Coordinar con los Gobiernos Departamentales y Municipales, incorporando a la población civil, mejoras a los espacios residenciales con alumbrado público e implementación de espacios comerciales así como recreativos en las zonas residenciales.

- Promover actividades de información y formación, no escolarizada, para la prevención del delito, incorporando la participación activa de Escuelas, Colegios, Universidades e Institutos.

- Implementar programas de aislamiento preventivo y re-socialización del delincuente. Para ello se requiere la participación activa de instituciones públicas, privadas y de la sociedad civil.

- Crear espacios públicos y participativos, para la discusión, desarrollo y evaluación de las políticas, estrategias y actividades relacionadas con la Seguridad Ciudadana. Estos espacios de debate deben realizarse en diferentes niveles, desde lo inter-institucional (instituciones públicas y privadas), a nivel de organizaciones y agrupaciones sociales, así como a niveles de comunidades territoriales (distritos y barrios).

- Incorporar al control interno policial a instituciones de la sociedad civil, especialmente organizaciones académicas, que contribuyan a la prevención y control de la corrupción.

\section{Conclusión}

La percepción de la legitimidad policial está determinada por la confianza en la policía, que en la actualidad se ha convertido en una percepción bastante volátil en relación a antaño, lo cierto es que la publicación de casos de malas prácticas y corrupción policial que trascienden en el 
debate público y político coinciden al mismo tiempo con valoraciones públicas relativamente positivas de la policía y los servicios policiales.

Percepciones ambivalentes que se deben por un lado al rol que tiene la institución policial en el mantenimiento del orden social que beneficia al conjunto de la ciudadanía, pero que no deja de ser un orden concreto y desigual que tienen que ver con la calidad de los medios e instrumentos utilizados para mantener ese orden.

La agudización del conflicto social en las últimas cuatro décadas como consecuencia del aumento de la desigualdad y del avance de la exclusión social en el marco de la globalización del capitalismo flexible ha obligado a las policías a intervenir en diferentes manifestaciones de dicho conflicto, frente al delito, huelgas, bloqueos, marchas, etc., que ha supuesto que las valoraciones sobre la eficacia policial -para qué sirve el trabajo policial—y sobre la justicia distributiva de sus acciones -a qué intereses sirve - hayan condicionado la percepción de su imagen, especialmente entre los grupos sociales más expuestos a las dinámicas de exclusión; por lo que se deben buscar mecanismos que permitan que la Policía Boliviana cumpla su mandato constitucional, al mismo tiempo que su institucionalidad no esté sometida al interés sectorial de los gobernantes temporales, por lo que se debe perfeccionar la normativa que reglamenta su accionar.

\section{REFERÊNCIAS}

Alvarez, A. E. (2005). El estado de la seguridad en América Latina. Cuadernos de Desarrollo Humano. PNUD El Salvador, 70-88.

Belaunde de Cárdenas, A. de. (2015). Seguridad Ciudadana y Gobiernos Locales : El Caso de la Municipalidad de Miraflores. Artículos Originales.

Chalmers, A. F. (2000). ¿ QUÉ ES ESA COSA LLAMADA CIENCIA?

Constantino, G. (2014). Las políticas de seguridad en Argentina: los límites de la autonomía 
policial. Aposta: Revista de Ciencias Sociales, 63, 1-26.

Frühling, H. (2012). América Latina y el Caribe Como medirla y como La eficacia de las politicas públicas de seguridad ciudadana en América Latina y el Caribe Como medirla y como.

Gabaldón, L. G. (2013). La reforma policial en Venezuela : Lecciones aprendidas e implicaciones para las políticas públicas de seguridad ciudadana. Espacio Abierto Cuaderno Venezolano de Sociología, 22(3), 667-679.

García Gallegos, B. (2013). Seguridad ciudadana y policía comunitaria en contexto de cambio político y social. Universitas, Revista de Ciencias Sociales y Humanas, (19), 49-72.

Hernández-Baqueiro, A., \& Suárez-González, A. (2015). Perspectivas éticas de la seguridad ciudadana en Chile y México.

Jaime, Ó., \& Torrente, D. (2017). Los desafíos de la Policía como actor político en España. Revista Española de Ciencia Política, 45, 147-172.

Medina Ariza, J. (2010). Políticas de seguridad ciudadana en el contexto urbano y prevención comunitaria - La experiencia anglosajona. Revista Electrónica de Ciencia Penal y Criminología, 02, 2-36.

Morles, V. (2002). Sobre la metodología como ciencia y el método científico: un espacio polémico. Revista de Pedagogía, 23(66), 16.

Newman, O. (1972). Defensible Space: crime prevention through urban design. New York: Macmillano.

Paez Murillo, C. A., Peón Escalante, I. E., \& Ramírez, Pedraza, Y. (2018). Contexto de la seguridad ciudadana en América Latina y el Caribe : revisión de literatura (2007 - 2017). Revista Científica General José María Córdova, 16(24), 83-106.

PNUD. (2018). Human Development Reports 2018. Retrieved from http://hdr.undp.org/en/2018-update 
Requena Hidalgo, J. (2016). La legitimidad policial y la colaboración ciudadana con la policía. InDret Revista Para El Análisis Del Derecho, 2, 3-42.

Robles Garrote, P., \& Rojas, M. del C. (2015). La Validación por jicio de expertos: dos investigaciones cualitativas en lingüistica aplicada. Revista Nebrija, 18, 16.

Ruiz Vasquez, J. C. (2003). Policía Exitosa, Policía Indolente: Nuevas tendencias en seguridad ciudadana. Desafios, 9, 109-137.

Sabino, C. (1992). El proceso de investigacion. Bueno Aires, Argentina.

Salas Torres, G. A. (2015). Aspectos principales del espacio urbano y la seguridad ciudadana desde la perspectiva de Bogotá. Revista Criminalidad, 2(57), 301-317.

Salgado Ibarra, J. (2010). Documento conceptual-metodológico sobre políticas públicas de seguridad ciudadana, capacidades institucionales para medir su desempeño y bases para el desarrollo de indicadores en esta materia.

Sozzo, M. (2012). Los retos de la izquierda en las políticas públicas de seguridad ciudadana.

Torres Angarita, A. (2010). La politica pública de seguridad ciudadana en Quito: un esfuerzo municipal. URVIO Revista Latinoamericana de Estudios de Seguridad, 9, 70-88.

Vidales Rodríguez, C. (2010). Seguridad ciudadana, políticas de seguridad y estrategias policiales. Revista de Estudios Penales y Criminológicos, XXXII, 22. 